\title{
Phase Stability Diagrams for Nanostructured Thin Film Multilayers
}

\author{
G.B. Thompson, R. Banerjee, and H.L. Fraser \\ Department of Materials Science and Engineering, The Ohio State University, 2041 College Road, \\ Columbus, Ohio 43210
}

As individual layers are reduced in thickness in a A/B multilayered thin film stack, pseudomorphic phases can be stabilized. The stabilization of these phases is a result of the competition between the volumetric and interfacial free energy. Recently a classical thermodynamic model has been proposed to model phase stability in such systems [1]. The total free energy per unit interfacial area, $\Delta g$, is given as

$\Delta g=\left[\Delta G_{A} f_{A}+\Delta G_{B} f_{B}\right] \lambda+2 \Delta \gamma$

where $\Delta G_{i}$ is the volumetric free energy difference between the pseudomorphic and bulk equilibrium states, $f_{i}$ is the volume fraction of $i, \lambda$ is the bilayer thickness equal to the layer thickness of A plus the layer thickness of $\mathrm{B}$, and $\Delta \gamma$ is the interfacial free energy reduction between the pseudomorphic and bulk equilibrium interfaces. Thus, volume fraction and $\lambda$ act as two independent degrees of freedom in stabilizing the pseudomorphic phase. A plot, referred to as a biphase diagram, of the inverse bilayer thickness, $\lambda^{-1}$, and the volume fraction, $f_{i}$, can be used to map out regions of phase stability. This provides a quick and convenient reference in which combinations of length scale and volume fraction would stabilize either the bulk equilibrium phase or the pseudomorphic phase. These phase stability plots have been referred to as biphase diagrams.

Lowe and Geballe [2] reported that $\mathrm{Zr}$ can under go a change in phase stability from $h c p$ to $b c c$ when the bilayer spacing is reduced below $3.1 \mathrm{~nm}$ for an equal layer thickness specimen. Based upon the classical thermodynamic model, additional $h c p$ to $b c c$ phase stability should exist for $\mathrm{Zr}$ as the volume fraction is varied. Using this data point as a reference, a series of Nb-rich volume fraction multilayers were sputtered deposited. Plan-view electron diffraction confirmed additional phase stability changes in the Zr layers. These have been plotted on the biphase diagram of Fig. 1 . A line intensity scan of the plan-view electron diffraction pattern of Fig. 2 revealed that upon a change in phase stability, the Zr layer adopted a lattice parameter very similar to its high temperature $b c c \beta-Z r$ phase indicated by the asymmetric $\{011\}$ intensity profile [3]. Upon further reduction in layer thickness for a fixed volume fraction, the $b c c \mathrm{Zr} / b c c \mathrm{Nb}$ interface became coherent.

Since $\mathrm{Zr}$ was stabilized as a pseudomorphic $b c c$ phase in the $\mathrm{Nb}$-rich volume fraction multilayer, could a $h c p \mathrm{Nb}$ phase be stabilized for a Zr-rich volume fraction multilayer. A series of Zr-rich volume fraction multilayers were sputtered deposited as shown in Fig. 1. Plan-view electron diffraction of multilayer \#10 on Fig. 1 can be seen in Fig. 3. This diffraction pattern was consistently indexed as two hcp phases [4]. The stabilization of the $h c p \mathrm{Nb}$ phase, similar to the $b c c \mathrm{Zr}$ phase, is a result of the interfacial energy reduction. These phases have been confirmed by transmission x-ray diffraction. This type of phase diagram provides a quick and convenient tool to scientists in modeling and predicting phase stability in multilayered thin films. 


\section{References:}

[1] S.A. Dregia, R. Banerjee, and H.L. Fraser, Scripta Mater. 29(2) (1998) 217.

[2] W. P. Lowe and T.H. Gaballe Phys. Rev. B 29(9) (1984) 4961.

[3] G.b. Thompson et al. Materials Research Society Spring Proceedings 727 (2001) 59.

[4] Gregory B. Thompson "Predicting Polymorphic Phase Stability in Multilayered Thin films" Ph.D. Dissertation The Ohio State University (2003).

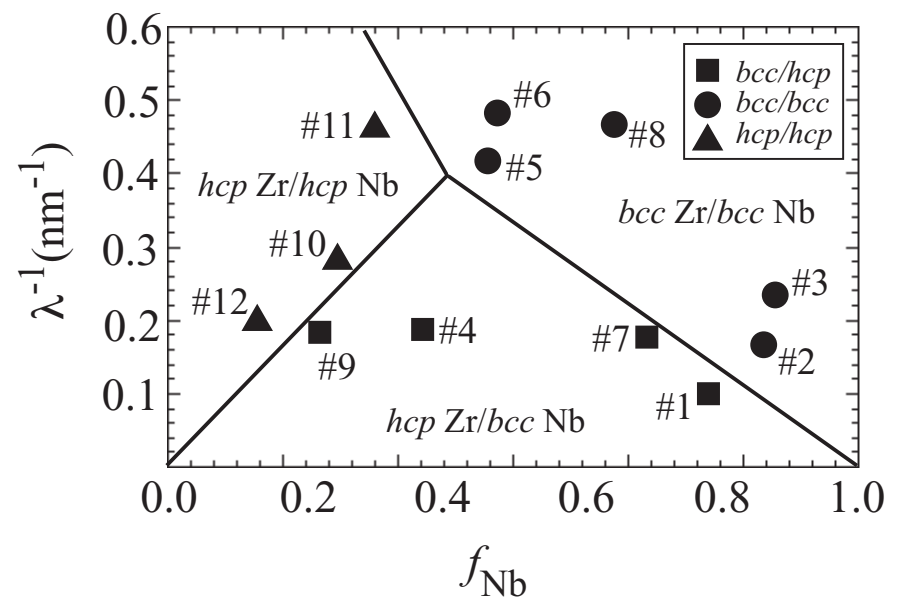

Fig. 1 Biphase diagram for $\mathrm{Zr} / \mathrm{Nb}$ multilayers [4].

$\# 1$

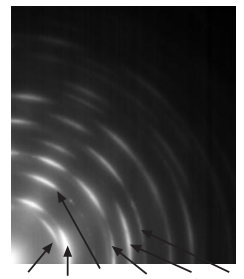

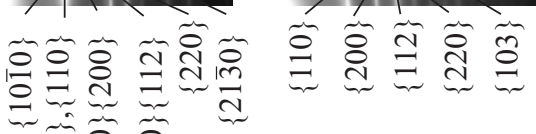

示陉
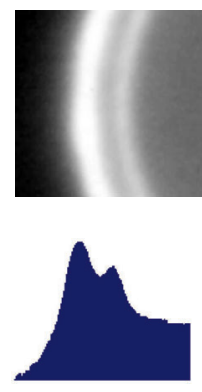

䓍
\#2
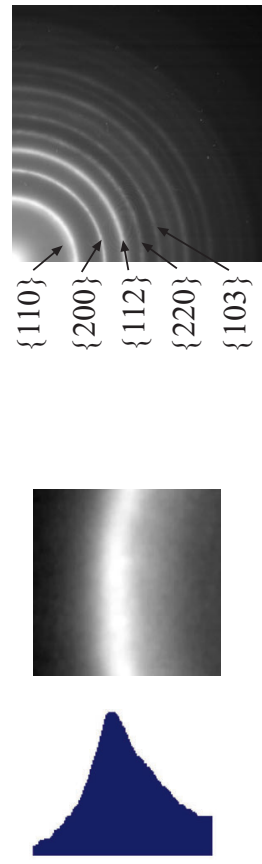

$\approx \sim$
$\Xi \approx$
$\approx \sim$
\#3

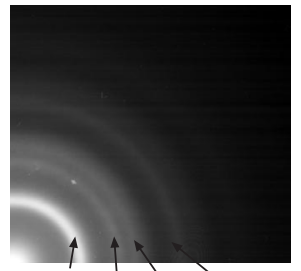

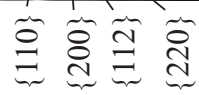
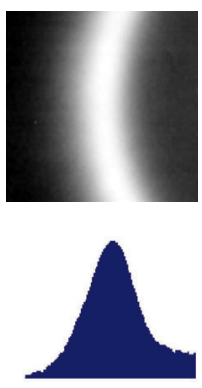

$\underset{\sim}{\stackrel{0}{\Xi}}$
$\{20 \overline{2} 0\} \mathrm{Nb}$

$\checkmark$

\section{$\{20 \overline{2} 0\} \mathrm{Zr}$}

$\{10 \overline{1} 1\} \mathrm{Zr}, \mathrm{Nb}$
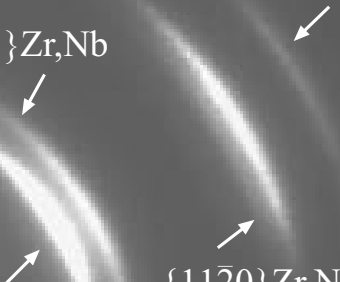

$\{10 \overline{1} 0\} \mathrm{Zr}, \mathrm{Nb}$

$\{11 \overline{2} 0\} \mathrm{Zr}, \mathrm{Nb}$

Fig. 3 Plan-view electron diffraction of hсp $\mathrm{Zr} /$ hcp $\mathrm{Nb}$ ( \#10) [4].

Fig. 2 Plan-view electron diffraction of $h c p-b c c$

Phase stability in $\mathrm{Zr}$ for $\mathrm{Zr} / \mathrm{Nb}$ multilayers [3]. 